\title{
La pena de prestación de servicios a la comunidad, como alternativa positiva y la creación de un registro nacional, para su efectiva aplicación
}

\author{
The penalty for the provision of services to the \\ community, as a positive alternative and the creation of a \\ national registry, for its effective application
}

\author{
Jesús Josué Ramos Falcón ' \\ Universidad Nacional Mayor de San Marcos \\ josue_ramo02@hotmail.com
}

Presentado: 16/03/2019 - Aceptado: 25/09/2019 - Publicación: 12/04/2021

\begin{abstract}
Resumen
Se estudia la pena de prestación del servicio a la comunidad en sus diversos componentes temáticos: Su regulación, grado de aplicación, desarrollo dogmático. Se examina sus ventajas en relación a los beneficios de rehabilitación, reeducación y resocialización del condenado. Entre las principales causas de su mínima aplicación destacan: el escaso desarrollo doctrinal; la imprecisa regulación normativa sobre la conversión, la falta de difusión como pena alternativa a la pena privativa de libertad. Finalmente es preciso señalar que la pena de prestación de servicios a la comunidad es más efectiva y productiva que la reserva del fallo condenatorio y la suspensión de la ejecución de la pena.
\end{abstract}

Pese a las modificaciones legislativas sobre la pena de prestación del servicio a la comunidad; es inminentemente la aplicación de un control y registro de estos condenados a través de la creación de un Registro Nacional de Sentenciados a la pena de prestación de servicios a la comunidad "RENSPSC" con el empleo de las herramientas virtuales. El Registro de estos condenados permitiría contar con información actualizada, en tiempo real, sobre las entidades receptoras, modalidades de cumplimiento de la pena, grado de cumplimiento; órganos jurisdiccionales emisores de la sentencia, entre otras variables.

Palabras clave: Prestación servicios a la comunidad; registro nacional.

\begin{abstract}
The penalty of providing the service to the community in its various thematic components is studied: its regulation, degree of application, dogmatic development. Its advantages are examined in relation to the benefits of rehabilitation, reeducation and resocialization of the convicted. Among the
\end{abstract}

(c) Los autores. Este artículo es publicado por Lucerna luris Et Investigatio de la Facultad de Derecho y Ciencia Política de la Universidad Nacional Mayor de San Marcos. Este es un artículo de acceso abierto, distribuido bajo los términos de la licencia Creative Commons Atribución 4.0 Internacional (CC BY 4.0) [https://creativecommons.org/licenses/by/4.0/deed.es] que permite el uso, distribución y reproducción en cualquier medio, siempre que la obra original sea debidamente citada de su fuente original. 
main causes of its minimal application are: poor doctrinal development; the imprecise regulatory regulation on conversion, the lack of dissemination as an alternative penalty to the custodial sentence. Finally, it should be noted that the penalty for rendering services to the community is more effective and productive than the reservation of the conviction and the suspension of the execution of the sentence.

Despite legislative changes on the penalty of providing the service to the community; It is imminently the application of a control and registration of these convicted through the creation of a National Registry of Sentenced to the penalty of providing services to the community "RENSPSC" with the use of virtual tools. The Registry of these convicted persons would allow to have updated information, in real time, on the receiving entities, modalities of compliance with the penalty, degree of compliance; Courts issuing the judgment, among other variables.

Keywords: Provision of community services; national register.

\section{Introducción}

El presente tema de investigación, se centra en dos aspectos; en la prestación del servicio a la comunidad; como alternativa positiva ; asimismo, en la creación de un registro nacional, considerando el uso de las nuevas tecnologías, para una efectiva aplicación de la misma.

Primero, nos enfocaremos en el replanteamiento de la política criminal actual, buscando un mecanismo alternativo diferente a la pena efectiva o de la reserva del fallo condenatorio o la suspensión de la ejecución de la pena. Por eso, parte de la investigación, se centra en la pena de prestación del servicio a la comunidad; a fin, de preferir otras formas de sanción menos gravosas, evitar la congestión carcelaria, la estigmatización del condenado y la inocua imposición de penas suspendidas con reglas de conducta pocas veces cumplidas. Asimismo, el beneficio de éste tipo de pena; es, la resocialización, la inserción del condenado a la sociedad. Cabe precisar; que la pena de prestación del servicio a la comunidad; se encuentra tipificado en el artículo 34 del Código Penal; además, conforme al artículo 32, establece que su aplicación puede ser autónoma, cuando específicamente se señala en cada delito; o también, puede ser sustituta o alternativa a criterio del juez, cuando la sanción no sea superior a cuatro años; cabe precisar, que cuando se aplique de manera sustituta a la pena privativa de libertad; será, de conformidad a lo establecido en el artículo 52, sobre conversiones de la pena.

Segundo, es respecto a la utilización de las nuevas tecnologías, para el desarrollo efectivo en la administración de justicia; en nuestro caso concreto, realizar una web service, denominado: Registro Nacional de sentenciados por prestación del servicio a la comunidad "RENSPSC", que será usado por la Policía Nacional del Perú, Ministerio Público, el Poder Judicial y el Instituto Nacional Penitenciario; cuya finalidad, es trabajar en cooperación interinstitucional; para llevarse a cabo, una política de aplicación efectiva 
de ésta pena, desarrollarse estrategias de confrontación con la delincuencia, tener una data de las personas condenadas por ésta pena, permite saber cuántos sentenciados realizan la prestación de servicios comunitarios, el periodo por realizarse, si son reincidentes o en el caso de incumplimiento; asimismo, generar estadísticas en boletines anules, un control y monitoreo por departamentos; sobre las personas condenadas por la pena de prestación del servicio a la comunidad.

\section{Antecedentes de la pena de prestación de servicios a la comunidad y sobre el registro informático usado por instituciones públicas}

En legislaciones comparadas, se basaron en la búsqueda de aplicar penas sancionadoras distintas a la pena privativa de libertad; es decir, que en sus respectivos Código Penales, de: España (1995), Brasil (1984), Francia (1994), Cuba (1987) y entre otros países; asimismo, nuestro Código Penal (1991).

A nivel nacional, un claro antecedente de aplicar penas sustitutas a la pena privativa de libertad; se da, con la condena condicional, que establecía el Código Penal (1924); consecutivamente se fue incorporando diferentes penas alternativas, logrando vigencia hasta nuestro actual Código Penal; y que entre éste tipo de penas alternativas, tenemos la pena de prestación del servicio a la comunidad. Por ello, en la Exposición de Motivos de nuestro Código Penal; señala: "a pesar de reconocer la potencia criminógena de la prisión, considera que la pena privativa de libertad mantiene todavía su actualidad como respuesta para los delitos que son incuestionablemente graves. De esta premisa se desprende la urgencia de buscar otras medidas sancionadoras para ser aplicadas a los delincuentes de poca peligrosidad, o que han cometido hechos delictuosos que no revisten mayor gravedad."

Frente a lo expuesto en líneas anteriores; lo que se busca, es el cumplimiento de la pena de prestación de servicio a la comunidad, como alternativa positiva para sentenciados de mínima peligrosidad, que en vez en cumplir una condena efectiva o suspendida o convertida; ésta aplicación sería óptimamente efectiva, con la aplicación de nuevas tecnologías, como: software, web, programas, aplicaciones, etc., que influye en el entorno social y personal, que son de uso instantáneo, práctico, seguro; y, puede ser de utilidad en los diferentes sectores públicos. Ejemplos que tenemos, de los órganos de administración de justicia, que usan las herramientas de la era digital, como: La Ley $\mathrm{N}^{\circ}$ 28970, publicado el 12 de julio del 2007, que crea el registro de deudores alimentarios morosos "REDAM", siendo el Órgano de Gobierno del Poder Judicial; cuya finalidad, es tener una información consolidada y registrar aquellas personas que tienen resolución judicial consentida o ejecutoriada de obligación alimentaria y que adeuden 03 cuotas sucesivas o no; ésta información se proporcionará a la SBS, a efectos, que registre en la Central de Riesgos de dichas instituciones y adicionalmente 
podrá remitirse a las centrales de riesgo privadas. También; el registro de deudores judiciales morosos "RDJUM", creado por el Decreto Supremo $\mathrm{N}^{\circ}$ 11-2014-JUS, publicado parcialmente el 27 de diciembre del 2014, en El Peruano; que contenía un registro de deudores que tienen resolución firme y no cumplan con el pago de su deuda, siendo un Convenio de Cooperación Interinstitucional, de la Superintendencia de Banca, Seguros y Administradoras Privadas de Fondo de Pensiones (SBS) y está a cargo del Consejo Ejecutivo del Poder Judicial, éste tendrá a cargo el diseño, desarrollo, implementación progresiva y mantenimiento del sistema informático. Ambos programas desarrollados a cargo del Poder Judicial en cooperación interinstitucional; sin embargo, existe otros dos programas que realizan un registro informático, como: el Registro nacional de detenidos y sentenciados a pena privativa de libertad efectiva "RENADESPPLE", creado por la Ley $\mathrm{N}^{\circ}$ 26295 y el Decreto Supremo $N^{\circ}$ 001-96-JUS, que lo reglamenta; cuya finalidad de éste registro nacional, es ubicar rápidamente a toda persona detenida a nivel nacional, así como, tener una base de datos de las personas sentenciadas con una pena privativa de libertad efectiva, conservándose la información y realizándose estadísticas periódicas, también incorpora en su base de datos a los menores infractores retenidos a nivel policial, fiscal y judicial; y lo que se busca con este registro, es ser una vía web service, que contenga la información de detenidos en tiempo real. Por último; tenemos la Ley $\mathrm{N}^{\circ} 30364$ "Ley para prevenir, sancionar y erradicar la violencia la violencia contra la mujer y los integrantes del grupo familiar", que mediante Resolución de la Fiscalía de la Nación $N^{\circ}$ 0002420-2018-MP-FN, aprueba el reglamento del Registro único de víctimas y personas agresoras "RUVA"; cuya finalidad, es tener un sistema intersectorial y que orienta la intervención del Estado en materia de prevención, atención, protección y reparación de la violencia contra la mujer o de cualquier integrante del grupo familiar, así también, en hacer un monitoreo y seguimiento de las personas que cometan dichos actos de agresión; dicho programa, es responsabilidad del Ministerio Público, en coordinación con la Policía Nacional del Perú, el Poder Judicial y el Ministerio de la Mujer y Poblaciones Vulnerables. Conforme a lo expresado líneas arriba, nos preguntaremos, ¿sí crear un registros de condenados por prestación del servicio a la comunidad, serviría para optimizar la aplicación de la prestación de servicios a la comunidad? , conforme al estudio realizado en el presente trabajo, se sustentará.

\section{Contexto Problemático}

\subsection{La informática y la posibilidad de sistematización de la pena de prestación de servicios a la comunidad.}

Actualmente, dentro de un mundo globalizado que día a día avanza en la denominada era de la revolución cibernética; por lo que, a nivel mundial, en los diferentes sectores sociales, culturales, económicos, en lo personal y entre otros aspectos, se ve influenciado por el desarrollo de la informática. 
Un ejemplo es el Internet, que sirve como un medio transnacional de comunicación; a su vez, permite el intercambio, elaboración y creación de la información mediante el uso de diversas modalidades de comunicación en los diferentes medios electrónicos.

"El avance de la informática en el mundo actual, es de tal magnitud que se ha llegado a sostener que se constituye en una forma de Poder social. Las facultades que el fenómeno informático pone o coloca a disposición de los gobiernos y de las personas naturales o jurídicas, con rapidez y ahorro consiguiente de tiempo y energía, configuran un panorama de realidades de aplicación y de posibilidades" (Salinas Siccha 2008, p. 1195).

"La irrupción del ordenador y la red informática en la vida del hombre moderno representan un giro significativo en el transcurso de su historia, que afectan de un modo positivo su realidad cotidiana. Este fenómeno informático permite fomentar la difusión de ideas entre los individuos, sin depender de la posesión de los medios de comunicación. Los beneficios de la informática pueden percibirse sin mayores explicaciones en el terreno del comercio, la comunicación, el transporte, la educación, la medicina, entre otras áreas" (Aboso y Zapata 2006, p. 5).

Apreciamos así que la informática se encuentra presente en un mundo globalizado, que influye en la vida moderna de la persona. Siendo así, permite el desarrollo y avance a nuevas tecnologías, nuevos medios de comunicación y como herramienta para la ejecución de las distintas tareas que se presente en la vida del ser humano.

"Lo cierto es que en la actualidad nos hallamos viviendo en plena <<era de la informática>>, en la que, cada vez más, sofisticadas calculadoras electrónicas, funcionales, fiables y de gran capacidad han invadido los ámbitos más diversos de las relaciones socioeconómicas. Seguramente pocas dimensiones de nuestras vidas no se ven afectadas, dirigidas o controladas por el ordenador, directa o indirectamente. Sectores como la banca, los seguros, los transportes, la educación, la bolsa, el tráfico aéreo y terrestre, las administraciones públicas, es decir, la sociedad en su conjunto, dependen, en gran medida, de las computadoras. A ellas se les encomienda no sólo el archivo y procesamiento de la información, sino, incluso, la adopción automática de decisiones, por lo que se han convertido en el caballo de trabajo del siglo XXI, generándose, así, dicha dependencia" (Riquert 1999, p. 23). 
"El advenimiento de las computadoras masivamente paralelas está produciendo una verdadera revolución en la informática; en el futuro inmediato veremos muchos cambios de enfoque sobre la forma de hacer computación e incluso sobre el sujeto mismo de la programación. Desarrollos operados en vida artificial tienden a indicar que, en el futuro cercano, quienes hagan la programación pueden llegar a ser los programas mismos, en el sentido de que quede establecida la evolución por selección natural como el mejor método para obtener programas que utilicen en forma óptima las máquinas masivamente paralelas" (Puricelli 1997, p. 186).

Es así; que la informática y la posibilidad de sistematización de la pena de prestación de servicios a la comunidad, promueve una alternativa positiva para sentenciados de mínima peligrosidad, por lo que, en la actualidad es muy poco aplicado; por lo que, se busca el aumento de ésta pena sustituta, usando herramientas de la era digital, en la búsqueda de soluciones a mediano y largo plazo, que vayan de la mano con el desarrollo de la tecnología. Es así; que consideramos, la creación de un control, con la implementación de un registro de personas sentenciadas por pena de prestación del servicio a la comunidad; es decir, que sería una herramienta de aplicación práctica, con capacidad de guardar una data, determinar su aplicación objetiva, mediante la inclusión de una serie de datos a un determinado programa sistema que guarde estos registros. En ese mismo enfoque, "las posibilidades del ordenador son inmensas, infinitas; permiten una verdadera enseñanza conforme a las ideas, las necesidades y lo que pretende el hombre, llegando a hacer muchas cosas como éste, pero a mucha velocidad y sin cansancio" (Núñez Ponce, 1996, p. 19).

Un claro ejemplo sobre el uso de las nuevas tecnologías, que están implementando las instituciones púbicas, que administran o cooperan con la administración de justicia, como: software, web, programas, aplicaciones, etc.; son: El registro de deudores alimentarios morosos "REDAM", el registro de deudores judiciales morosos "REDJUM", el Registro nacional de detenidos y sentenciados a pena privativa de libertad efectiva "RENADESPPLE", el Registro único de víctimas y personas agresoras "RUVA"; por lo que, la creación de un Registro Nacional de sentenciados por prestación del servicio a la comunidad "RENSPSC", permitiría ésta herramienta en nuestra era digital: una información consolidada y detallada de las personas que son sentencia por éste tipo de penas, una data de registro biométricos en cooperación interinstitucional, monitoreo y seguimiento de las personas sentenciadas por éste tipo de penas; siendo así, sería posible optimizar el uso de la prestación de servicios a la comunidad que realicen los sentenciados por este tipo de penas. Por lo que; se busca indagar en soluciones que no generen más gastos al Estado, que constituyan un avance en la lucha contra los delitos y faltas al ordenamiento jurídico; y que se retribuya a la sociedad. 


\subsection{La realidad problemática de la aplicación de la pena de servicios a la comunidad en nuestro sistema penal}

Respecto a nuestro tema de investigación, la realidad problemática se centra en el uso excesivo de la pena privativa de libertad efectiva, olvidando que sólo se usa como última ratio o última medida; existiendo otras medidas o mecanismo alternativos, que permiten reeducar y resocializar al sentenciado por su delito cometido; en otras palabras, no sólo se busca que cumpla con una pena por la conducta culpable, antijurídica y típica en el la norma, sino que mediante la prestación del servicio a la comunidad, siendo una pena alternativa; el sentenciado por este delito, contribuye en cierta forma en resarcir a la sociedad. Sin embargo, a la fecha no es muy frecuente utilizar estas medidas alternativas y en nuestra actualidad; aún sigue de moda, la tendencia de aplicar la pena privativa de libertad.

Sin embargo, la alternativa del presente trabajo de investigación, considera, que debe comenzar a cambiar por otros sustitutos penales. Por lo que, citando al maestro italiano Baratta, cuando hacía referencia a: "Derribar los muros de la cárcel, tiene para la nueva criminología el mismo significado pragmático que el derribar los muros del manicomio para la nueva psiquiatría". Es decir; que ya reflejaba sobre la urgencia de buscar medidas alternativas a la pena privativa de libertad, porque los centros penitenciarios, aparte de encontrarse sobre poblados, no permite al sentenciado que cumpla con el fin de la pena.

En el desarrollo de la ciencia penal, ha influido diferentes criterios garantistas y humanitarios; sin embargo, a pesar existir la necesidad de plantear nuevas modalidades o sustitutos penales, que hicieran de la pena una consecuencia jurídica ventajoso para el sentenciado, para el mismo Sistema Jurídico Penal y la sociedad, respetando in extensu los derechos fundamentales de la persona y protegiendo la equidad, en relación a la culpabilidad del sujeto imputado y los fines de la pena de reeducar, rehabilitar y resocializar. En tal sentido, como el doctor Prado Saldarriaga (1993, p. 44), refiere que la ejecución de las distintas penas privativas de libertad fue organizada en base al régimen alburiano; pero, al verse las deficiencias de infraestructura en los centros penitenciarios y ver que el Estado no podía cambiar el comportamiento del sentenciados, sólo con la aplicación de una pena; es que, nuestro cuerpo normativo en base a las legislaciones comparadas, acogiendo principalmente del proyecto alternativo alemán de 1966; es que, en 1969, nuestro país promulga el Decreto Ley 17581, que unifica el procedimiento de ejecución de penas; asimismo, regula como reglas el trabajo comunitario y programas de educación para los internos en centros penitenciarios.

Por ello, en la promulgación de nuestro actual Código Penal de 1991, sigue la línea de buscar medidas alternativas de la pena privativa de libertad; 
por lo que, en la exposición de motivos, precisa: "La Comisión revisora, a pesar de reconocer la potencia criminógena de la prisión, considera que la pena privativa de libertad mantiene todavía su actualidad como respuesta para los delitos que son incuestionablemente graves. De esta premisa se desprende la urgencia de buscar otras medidas sancionadoras para ser aplicadas a los delincuentes de poca peligrosidad o que han cometido hechos delictuosos que no revisten mayor gravedad. Por otro lado, los elevados gastos que demandan la construcción y sostenimiento de un centro penitenciario, obligan a imaginar nuevas formas de sanciones para los infractores que no amenacen significativamente la paz social y la seguridad colectivas".

Ante lo expuesto, nuestro ordenamiento jurídico busca que las medidas alternativas a la pena privativa de libertad, establecidas en el Código Penal, tales como: "La suspensión de la ejecución de la pena, la reserva del fallo condenatorio, las exenciones, las conversiones"; son alternativas del ordenamiento jurídico penal, ante el eminente aumento de la pena privativa de la libertad.

Por ello; el presente tema de investigación ve a la pena de prestación del servicio a la comunidad, regulado en el artículo 34 del Código Penal, como un trabajo gratuito que debe realizar el sentenciado; ya sea, en entidades públicas o privadas, dicha supervisión y control de la ejecución, está a cargo del Instituto Nacional Penitenciario - INPE. Dicha pena, puede imponerse de manera autónoma o como alternativa a la pena privativa de libertad. Asimismo, puede aplicarse en los casos que se impone como regla específica, para cuando se suspende la ejecución de la pena.

Coincidimos con la opinión de Prado Saldarriaga, cuando dice: "que las ventajas penales de este tipo de pena son destacadas con relevante amplitud por la doctrina. En particular se realza su potencialidad resocializadora y la ausencia de efectos estigmatizantes." Sin embargo, existe cierta posición contraria al uso de medidas alternativas a la pena privativa de libertad, por considerarse muy pro derechos de los sentenciados o por considerar que en el sentenciado no surte efectos en el cambio de sus conductas; por lo que, desde mi perspectiva conservan el modelo penal del siglo XIX, sobre los fines de la pena, cuando no se consideraba que el sentenciado aparte de cumplir con la sanción penal impuesta por la vulneración del bien jurídico protegido, también debía cumplirse con la reeducación y resocialización del sentenciado después de cumplir con su condena impuesta.

El fundamento de la pena de prestación de servicios a la comunidad, es que sirve como una sanción diferente a la privación de libertad y agotadas estos mecanismos recién imponer la pena privativa; asimismo, el beneficio de prestación de servicios a la comunidad, viene hacer una pena coherente con la finalidad de la pena, cual es la resocialización, la inserción del condenado 
a la sociedad; es decir, esta forma de sanción coadyuva eficazmente en la resocialización del sentenciado; sin embargo, consideramos que para su correcta aplicación, debe hacerse una modificatoria parcial, en los artículos que regula ésta pena, como: el artículo 34 del Código Penal, sobre la prestación del servicio a la comunidad, a fin de ser efectiva su aplicación, considerando las aptitudes del condenado, los casos reincidencia y habitualidad; modificatoria parcial, de los artículos 52, 52-A, 53 y 55 del Código Penal; sobre la conversión de la pena privativa de la libertad, con el fin, que sea proporcional y de obligatoriedad en su aplicación; también, de los artículos 243 inciso 2 y 248 del Reglamento del Código Ejecución Penal; a fin, de recoger recomendaciones de organismos internacionales y crear un programa nacional, que haga efectivo la aplicación y cooperación interinstitucional.

Ahora bien, una de las consecuencias directas, están las cárceles con sobrepoblación penitenciaria, la inmensa mayoría de ellas con deficiencias infraestructurales, falta de alimentación para los internos, escasos recursos de personal penitenciario o de materiales para su reeducación o de programas que promuevan su rehabilitación; por lo que, el Estado Peruano, no puede abastecerse con los internos, considerando que existe entre procesados y sentenciados, a fin, de que cumplan con los fines de la pena .

Por ello; la pena de prestación del servicio a la comunidad, es una alternativa positiva en nuestra legislación penal peruano; es importante; porque, es uno de los temas menos tratados y aplicados por los jueces; que si bien es cierto, para su efectiva aplicación, deben hacerse algunas modificatorias en su cuerpo normativo. Sin embargo; para los penados que hayan cometido delitos o faltas de mínima gravedad o peligrosidad, se les debe dar una segunda oportunidad, que les ayude a resarcir el daño y contribuir con la sociedad, al igual que se reeducará, rehabilitará y permitirá su reinserción. Por eso; se desarrolla nuestra investigación, porque persigue en parte, el replanteamiento de la pena de prestación del servicio a la comunidad; a fin, de propiciar una aplicación racional, justa y hacer un uso frecuente y efectivo de esta pena

\subsection{Propuestas para la pena de prestación de servicios a la comunidad, como alternativa positiva y la creación de un registro nacional}

Posteriormente, después de analizar el desarrollo normativo y sobre la importancia del desarrollo de las nuevas tecnologías, en el uso de la administración de justicia; evaluamos en que debe existir una reforma en la pena de prestación del servicio a la comunidad. Considerando que nuestro Código Penal, respecto a la prestación del servicio a la comunidad, puede aplicarse de manera autónoma o sustituta; es así, que se propone (7) siete modificatorias; de las cuales, la Primera propuesta, es complementar el inciso 3 del artículo 34 , en vez de decir "Los servicios son asignados, en lo posible, conforme a 
las aptitudes del condenado..." debiendo establecer "conforme a habilidades personales, técnicos o profesionales en la función que desempeñen.... Segunda propuestas, en concordancia del mismo inciso 3 del artículo 34, cuando refiere: “... debiendo cumplirse en jornadas de diez horas semanales, entre los días sábados, domingos o feriados, de modo que no perjudiquen la jornada normal de su trabajo habitual." debería tipificar lo siguiente "...debiendo cumplirse un día de jornada laboral con un mínimo de dos horas diarias o cumplir doce horas máximo semanal, de modo que no perjudique la jornada normal de su trabajo habitual". Tercera propuesta, implementar un inciso más al artículo 34, por lo que sería "34.5 - No se aplica en casos de Reincidencia o Habitualidad; en base a los artículos 46-B y 46-C, respectivamente". Cuarta propuesta, es correspondiente al Reglamento del Código Ejecución Penal, en su artículo I del Título de Preliminar: Ámbito de Aplicación del Código Ejecución Penal, cuando refiere, que: "la administración, supervisión, y control de la ejecución de las penas de prestación de servicio a la comunidad y limitación de días libres, está bajo la Dirección de Tratamiento del INPE"; por lo que, en relación a su reglamento nos referimos al artículo 243 inciso 2 , que describe: “...INPE, celebrará convenios de cooperación institucional con los representantes de las municipalidades y la Policía Nacional, en aquellas provincias, distritos y localidades donde el INPE no cuente con oficinas de la Dirección de Tratamiento de la correspondiente Dirección Regional." Por lo que debería modificarse; ya que, con el D. Leg. N 1191 agregó en el artículo 34.2 del Código Penal, que puede ejecutarse en instituciones privadas y ello no se refleja en el Reglamento del mismo código; por lo que debería precisar lo siguiente: "INPE, celebrará convenios de cooperación institucional con los representantes de las municipalidades y la Policía Nacional; asimismo, de igual forma podrá celebrar convenios con otras instituciones públicas o privadas...". Quinta propuesta, en el reglamento en su artículo $248^{\circ}$, que describe: “...se considera prestación del servicio a la comunidad todo trabajo voluntario, gratuito, personal y de utilidad pública." Por lo que, debería agregarse, “...se considera prestación del servicio a la comunidad todo trabajo voluntario, gratuito, personal, de utilidad pública; a fin, de que el penado pueda rehabilitarse de manera satisfactoria." Sexta propuesta, la modificatoria de los artículos del Código Penal, respecto al Artículo 52 - Conversión de la pena privativa de libertad, artículo 52-A - Conversión de la pena privativa de la libertad en ejecución y del artículo 53 numeral 2 - Revocatoria de la conversión; en razón que establece: "Una jornada de servicio a la comunidad o una jornada de limitación de días libres, por cada siete días de pena privativa de libertad. "Debiendo ser lo siguiente: "Un día de prestación de servicio de la comunidad o un día de limitación de días libres, sustituye un día de pena privativa de libertad." Si se emplea dicha proporción se alcanzará un cumplimiento más coherente con la función asignada a las penas sustitutas. Séptima propuesta, sería crear un Registro Nacional de sentenciados por prestación del servicio a la comunidad 
"RNSPSC", encargado de realizar reuniones de trabajo en cooperación , con las instituciones involucradas en el tema, como: el Poder Judicial, Instituto Nacional Penitenciario y las entidades receptoras (Policía Nacional del Perú y el Ministerio Público); dichas instituciones, tomarán acuerdos para que se involucren y viabilicen la aplicación de la presente pena; cuya finalidad, es llevar a cabo una política de aplicación efectiva de ésta pena, desarrollar estrategias de confrontación, tener una data de las personas condenadas por ésta pena, saber el tipo de prestación de servicio a la comunidad que realizará, el periodo por realizarse, si son reincidentes o en el caso de incumplimiento; asimismo, generar estadísticas en boletines anules y hacer un mapeo por departamentos; sobre las personas condenadas por la pena de prestación del servicio a la comunidad. Por lo expuesto e investigado, de allí las propuestas planteadas en la presente investigación.

\section{Conclusiones}

El presente tema, se centró en dos aspectos, en el replanteamiento de la norma jurídica; respecto, a la prestación del servicio a la comunidad como alternativa positiva; y asimismo, en un segundo aspecto, sobre la creación de un registro nacional, para una efectiva aplicación de la misma.

Como ya dejamos expresado, en nuestra investigación, el desarrollo y la aplicación de la prestación de servicios a la comunidad como alternativa positiva, frente a la pena de privación de la libertad o una pena suspendida o de reserva de fallo condenatorio; asimismo, apreciamos que la era digital y tecnológica, se encuentra presente en todo los sectores sociales, siendo un mundo globalizado, que desarrolla la persona en su día a día ; es decir, que se encuentra en todas las ramas del saber humano, con progresos tecnológicos constante; es así que, instituciones públicas, que ejercen la administración de justicia o de aquellas que colaboran de manera conjunta; utilizan éstas herramientas, para crear una data o registro; así también, como ejecutar otras tareas pertinentes al desarrollo institucional y social.

En ese sentido se ha realizado un adecuado estudio histórico y doctrinario de la prestación de servicios a la comunidad y de la importancia de la informática, con un papel protagónico en la era digital; concluyendo con presentar siete propuestas, entre ellas, para replantear el cuerpo normativo, para su correcta regulación de la pena de prestación del servicio a la comunidad; y para efectivizar el mismo, la creación de una web service que éste a disposición de los órganos que administren o cooperen con la administración de justicia; como: registro de personas sentenciadas que se encuentran cumpliendo condenas por esta pena.

Es así; que el presente trabajo busca replantear el uso de la pena de prestación de servicio a la comunidad, considerando, que el uso de la tecnología, es indispensable en nuestro país y las propias instituciones 
públicas y privadas, no son ajenas al uso de éstas herramientas electrónicas y/o digital; por lo que, así como la pena de prestación de servicios a la comunidad, se basada en los principios modernos penales, orientados a la protección de la persona humana, éstos también, deben estar monitoreados o regulados con el uso de un registro o data de personas condenadas por prestación del servicio a la comunidad.

Asimismo, que el presente estudio, abre al debate sobre, como aplicar y regular éste tipo de pena; de replantear los conceptos enseñados por el docente, el legislativo en promover medidas alternativas sobre el uso de la pena privativa de libertad; y sobre, la doctrina que en lo presente es muy escaso, respecto al tema. Por lo que, se requiere un mayor estudio y sea debatido para que forme parte del conocimiento de los alumnos, profesores y operadores, tanto legislativos como judiciales. Recordar, que la comisión revisora que dio origen a nuestro actual Código Penal, el mismo que buscó que se aplique medidas alternativas sancionadoras, que resulten ser positivas para el sentenciado de mínima peligrosidad o que no sean reincidentes o habituales en cometer hechos delictuosos; y así, darles una oportunidad y que se cumpla con los fines de la pena, respecto a la rehabilitación, reeducación y resocialización. A su vez, para efectivizar su aplicación, se necesita del uso de nuevas tecnologías, que sirva para monitorear y llevar un control; que más utilidad, que apelar a los avances que nos brinda la modernidad, y propiciar la utilización de la informática como un medio técnico de avanzada sistematización y aplicación generalizada de la pena de servicios comunitarios.

Por último; para la aplicación de la pena de prestación de servicios a la comunidad, el Estado deberá realizar en relación a su marco institucional y las necesarias modificaciones legislativas; ya que, se pretende hacer una mejora de la legislación en armonía con la política criminal preventiva, protectora y garantista. En ese sentido, debe ir de la mano con la utilización de las herramientas de la era digital, para mejorar el desarrollo del uso de la prestación de los servicios comunitarios; siendo, un soporte técnico e informático, que almacene una base de datos, como la propuesta presentada, del Registro Nacional de sentenciados por prestación del servicio a la comunidad "RENSPSC", será usado por la Policía Nacional del Perú, Ministerio Público, el Poder Judicial y el Instituto Nacional Penitenciario, trabajando en cooperación interinstitucional; para llevarse a cabo, una política de aplicación efectiva de ésta pena; cuya finalidad, es tener un control biométrico y monitoreo del condenado, saber el tipo de prestación de servicio a la comunidad, el periodo por realizarse y en el caso de incumplimiento de la misma.

\section{Referencias Bibliográficas}

Aboso, Gustavo Eduardo y zapata, María Florencia (2006). Cibercriminalidad y Derecho Penal. Editorial IB de f. de Montevideo - Buenos Aires. Julio César Faira Editor. Buenos Aires. 
Baldova Pasamar, M. A. (1998). Penas privativas de Derechos. En lecciones de consecuencias jurídicas del delito. Gracia Martín, Luis (coordinador). Editorial Tirant lo Blanch. Valencia - España.

Beccaria, Cesare (1980). De los Delitos y de las Penas. Biblioteca Aguilar. Ediciones española. Barcelona

Berdugo Gómez De La Torre, Ignacio (1993). Temas de Derecho Penal. Editorial Cultural Cuzco. Lima - Perú.

Hugo Vizcardo, Silfredo (2010). Delitos Contra el Patrimonio. Pro Derecho Perú Investigaciones Jurídicas. Lima - Perú.

Hurtado Pozo, José (2005). Manual de Derecho Penal, Parte General. $3^{\text {a }}$ Edición. Editorial Grijley. Lima - Perú.

Mir Puig, Santiago (2002). Introducción a las bases del Derecho penal. Concepto y método. 2da Edición. Julio César Faiora Editor. Montevideo.

Mir Puig, Santiago (2004). Derecho Penal. Parte General. 7ma edición. Editorial B de F. Julio César Faira Editor. Montevideo.

Muñoz Conde, Francisco (2000). Derecho Penal, Parte General. 4⿳a Edición, Revisada. Tirant lo Blanch. Barcelona.

Nuñez Ponce, Julio César (1996). Derecho Informático, nueva disciplina jurídica para una sociedad moderna. Editorial Marsol. Trujillo - Perú.

Peña Cabrera, Raúl (1994). Tratado de Derecho Penal. Estudio Programático de la Parte General. Lima: Editorial Grijley. Lima - Perú.

Peña Cabrera Freyre, Alonso Raúl (2009). Derecho Penal. Parte General. Teoría del delito y de la pena y las consecuencias jurídicas. Editorial Rodhas. Lima - Perú.

Prado Saldarriaga, Víctor Roberto (2000). Consecuencias Jurídicas del Delito en el Perú. $1^{\underline{a}}$ Edición. Gaceta Jurídica. Lima - Perú.

prado saldarriaga, Víctor (1993). Comentarios al Código Penal de 1991. Editorial Alternativas. Lima - Perú.

Puricelli, José L. (1997). Informática y delito. En Derecho penal y Derecho procesal penal. Homenaje a Carlos Alberto Contreras Gómez. Abeledo Perrot. Buenos Aires - Argentina.

Riquert, Marcelo A. (1999). Informática y derecho penal argentino. Ad - Hoc. Buenos Aires Argentina.

Salinas Sicha, Ramiro (2008). Derecho Penal Parte Especial. $3^{\circ}$ Edición. Editora Jurídica Grijley. Lima - Perú.

Villa Stein, Javier (2014). Derecho Penal, Parte General. Ara Editores E.I.R.L. Lima - Perú.

Villavicencio Terreros, Felipe (2005). Derecho Penal, Parte General. Editorial Grijley. Lima Perú.

\section{Notas al final}

1 Biografía: Abogado por el Colegio de Abogados de Lima, Perito por el Colegio Nacional de Peritos en Accidentología Vial. Egresado de la Maestría en Ciencias Penales, por la UNMSM; actualmente, ejerzo la formación académica y profesional. 
